ISSN 1997-342X (Online), ISSN 1991-8631 (Print)

Original Paper http://ajol.info/index.php/ijbcs

http://indexmedicus.afro.who.int

\title{
Influence de l'occupation des terres sur la dynamique des communautés végétales en zone Sahélienne : cas de la commune rurale de Dantchandou (Niger)
}

\author{
Rahila MAAZOU ${ }^{1 *}$, Habou RABIOU ${ }^{2}$, Youssoufa ISSIAKA ${ }^{1,4}$, Lawali ABDOU ${ }^{2}$, Sani \\ IDI SAIDOU $^{2}$ et Ali MAHAMANE ${ }^{2,3}$ \\ ${ }^{I}$ Institut Supérieur en Environnement et Ecologie, Université de Diffa, Niger. \\ ${ }^{2}$ Faculté des Sciences Agronomiques, Université de Diffa, Niger. \\ ${ }^{3}$ Faculté des Sciences et Techniques, Université Abdou Moumouni de Niamey, Niger. \\ ${ }^{4}$ Faculté d'Agronomie et des Sciences de l'Environnement, Université Dan Dicko Dankoulodo de \\ Maradi, Niger. \\ *Auteur correspondant ; E-mail :rahilamaazou@yahoo.fr
}

\section{REMERCIEMENTS}

Au Centre National de Surveillance Ecologique à Long Terme (CNSEE), du Niger, pour le financement de la mission de terrain de cette étude.

\section{RESUME}

La commune de Dantchandou est l'une des principales sources d'approvisionnement en bois de l'Ouest du Niger, ce qui engendre du coup une désorganisation de la structure et de la composition de paysage. L'objectif de la présente étude est d'évaluer l'impact de mise en culture, des pâturages, des jachères et l'influence des villages sur la dynamique des communautés végétales de la commune de Dantchandou. Les données ont été collectées dans les différentes unités d'occupations des terres (replat, versant, dépression et piedmont des plateaux) dans lesquelles sont délimitées des placettes de $1000 \mathrm{~m}^{2}$ en tenant compte de l'homogénéité de la végétation à fin d'éviter l'effet de lisière. Ainsi pour la caractérisation de la végétation trois transects ont été retenus. Un total de 76 relevés a été réalisé. L'étude a permis d'inventorier 151 espèces végétales dont 125 espèces herbacées et 26 espèces ligneuses réparties dans 101 genres et 28 familles. Dans les champs et jachères, 106 espèces ont été recensées réparties dans 81 genres et 23 familles. Dans les jachères, le cortège floristique est composé de 58 espèces réparties dans 47 genres et 17 familles. Sur les plateaux il a été recensé 111 espèces reparties en 24 familles. L'indice de diversité de Shannon est plus élevé dans les jachères avec 5,16 bits suivis des champs avec 5,08 bits et enfin sur les plateaux avec 5,05 bits. Cette diversité paysagère est lié aux différents types d'utilisation des terres, raccourcissement de la durée des jachères, expansion des agro-systèmes, le surpâturage avec comme conséquence la substitution des espèces autochtones par des espèces invasives.

(C) 2016 International Formulae Group. All rights reserved.

Mots clés : Occupation des terres, dynamique de communautés végétales, zone aride, Niger. 


\title{
Influence of land use on the dynamics of plant communities in the Sahelian zone: case of the rural commune of Dantchandou (Niger)
}

\begin{abstract}
The commune of Dantchandou is one of the main sources of wood supply in western Niger, causing a disorganization of the structure and composition of the landscape. The objective of this study is to evaluate the impact of cultivation, pasture, fallow land and the influence of the villages on the dynamics of the plant communities of the commune of Dantchandou. The data were collected in the different land use and land cover units (flat, slope, depression and foot of the plateaus). Plots of $50 \mathrm{~m}$ x $20 \mathrm{~m}$ were set in each sampling site taking into account the homogeneity of the vegetation in order to avoidedge effect. Thus, for the characterization of the vegetation, three transects were retained. A total of 76 surveys were conducted. Our results show an inventory of 151 plant species including 125 herbaceous species and 26 woody species distributed among 101 genera and 28 families. In fields and fallows 106 species were recorded which belong to 81 genera and 23 families. In the fallows the floristic composition is of 58 species from 47 genera and 17 families. On the plateaus 111 species belong to 24 families were recorded. The Shannon diversity index is higher in fallows with 5.16 bits followed by fields with 5.08 bits and finally on plateaus with 5.05 bits. This landscape diversity is linked to the different types of land use, shorter fallow periods, expansion of agrosystems, overgrazing resulting in the substitution of indigenous species by invasive species.
\end{abstract}

(C) 2016 International Formulae Group. All rights reserved.

Keywords: land use, dynamics of plant communities, arid zone, Niger.

\section{INTRODUCTION}

En zones arides et semi-arides, particulièrement le Sahel, les écosystèmes sont caractérisés par une végétation principalement de graminée annuelle parsemée des plantes ligneuses et d'une faible productivité (Cheula, 2009). Cependant, ces ressources vivement sollicitées, subissent depuis plusieurs décennies une forte dégradation qui entraîne des dysfonctionnements des écosystèmes terrestres et des pertes de biodiversité (FAO, 2003); encore amplifiés par les modes et systèmes inappropriés d'exploitation des ressources disponibles. Ces changements ont des répercussions directes sur l'occupation du sol et sur la dynamique des communautés végétales. Les processus naturels de succession des végétations sont alors perturbés par l'activité anthropique à travers l'exploitation du bois, aux différents systèmes d'exploitation de l'espace, en particulier aux systèmes de culture et/ ou d'élevage traditionnels et extensifs (Fournier et al., 2001). Cet état de fait est aisément perceptible dans les pays comme le Niger où l'économie est fondée sur le système agro-sylvo-pastoral (Ichaou, 2000).

Le paysage apparait comme le niveau convenable d'explication $\mathrm{du}$ processus écologique en cours car c'est une représentation de l'espace d'utilisation des terres. Selon Ichaou (2000), plus de 80\% de la surface de la Terre est directement influencée par les activités humaines qui modifient profondément les contraintes écologiques au sein des habitats, la distribution des habitats dans le paysage ainsi que le fonctionnement des populations et leurs interactions (Pascal, 2010). Ces perturbations se traduisent par le développement de défrichement illégal, de pastoralisme illégal dont les conséquences sont la disparition de la couverture végétale et de la biodiversité. Dans ce contexte de forte pression anthropique et de changement climatique, il est urgent d'évaluer la dynamique des communautés végétales. Il s'agit d'investiguer le processus actuel de dégradation accélérée du capital productif. Cette étude a pour but à partir des relevés de 
végétation de faire une analyse de la dynamique des communautés végétales qui permettrait de mieux comprendre les processus et dynamiques environnementales, paysagères que connaissent les écosystèmes dans la commune de Dantchandou ainsi que les enjeux liés à leur système multi usage dans un contexte global de l'occupation des sols afin d'apporter des éléments de réponse et de réflexion sur ces disparités spatiales.

\section{MATERIEL ET METHODES Zone d'étude}

La commune de Dantchandou est située à $75 \mathrm{~km}$ au Nord-Est de la ville de Niamey (capitale du Niger) entre $13^{\circ} 21^{\prime}$ et $13^{\circ} 42^{\prime}$ de longitude Est et $2^{\circ} 28^{\prime}$ et $2^{\circ} 52^{\prime}$ de Latitude Nord (Figure 1). La commune de Dantchandou appelée autrefois Fakara pour désigner le pays des plateaux situés en rive droite du Dallol Bosso, couvre une superficie d'environ $916,6 \mathrm{~km}^{2}$ (carte de la commune de Dantchandou, 2012). C'est la commune la plus orientale du département de Kollo dans la région de Tillabéri.

La commune de Dantchandou présente une température annuelle moyenne de $29,2{ }^{\circ} \mathrm{C}$ et une précipitation moyenne annuelle de $477 \pm 126 \mathrm{~mm}$. La végétation de la commune de Dantchandou est constituée essentiellement par des fourrés à Combretaceae sur les plateaux latéritiques, steppes sur les terrasses sableuses, dans les vallées sèches et sur les dunes fixées. La famille des Combrétacées y est fortement représentée et domine les plateaux. La structure arbustive est à dominance de Guiera senegalensis, Combretum micranthum, Combretum nigricans. On y trouve également d'autres essences comme: Annona senegalensis, Piliostigma reticulatum, Boscia angustifolia. La strate herbacée est dominée entre autres par Zornia glochidiata, Eragrostis tremula, Cenchrus biflorus, Aristidas pp. Brachiaria pp. (Saadou, 1990).

La population de la commune de Dantchandou est loin d'être répartie uniformément sur les terres cultivables. Elle est concentrée sur les terres les plus fertiles et les mieux arrosées (Saadou, 1990). L'accroissement démographique rend difficile ou impossible la pratique des jachères longues traditionnelles. Le problème d'une transformation des façons culturales et du système de production des céréales n'a pas évolué depuis des siècles. L'étude diachronique menée par (Ouédraogo, 2006; Mahamane et al., 2007; Morou, 2010) dans le terroir de Dantchandou à mis en évidence une dynamique interactive PopulationEnvironnement très marquée. En espace d'un demi-siècle, le paysage dans cette zone d'étude s'est complètement transformé : $12 \%$ de la surface totale sous l'emprise des cultures en $1950,35,3 \%$ en 1975 et $70,5 \%$ en 1992 (Morou, 2010).

\section{Collecte des données}

Un échantillonnage axé sur des transects suivant les types d'occupation des terres et de la géomorphologie a été effectué. Les transects sont placés depuis les sommets des plateaux jusqu'au bas fond en direction des villages (le plus souvent situés dans le bas fond). Dans chaque unité d'occupations des terres (replat, versant, dépression et piedmont des plateaux) est délimitée des placettes de 20 $\mathrm{m} \times 50 \mathrm{~m}$ soit $1000 \mathrm{~m}^{2}$ en tenant compte de l'homogénéité de la végétation ligneuse et herbacée afin d'éviter l'effet de lisière (Gounot, 1969). Ainsi pour caractériser la végétation ligneuse et herbacée trois transects sont retenus: transect 1 qui correspond au nord, transect 2 qui correspond au centre et le transect 3 qui correspond au Sud afin d'établir une liste floristique pour la commune de Dantchandou. Les relevés phytosociologiques ont été réalisés selon la méthode sigmatiste de Braun-Blanquet (1932) sur la base de l'homogénéité floristique et écologique des stations (Gounot, 1969). Un total de 76 relevés a été réalisé. Lors des relevés, sur une fiche normalisée sont notés les paramètres stationnels (coordonnées géographiques, texture du sol par la méthode tactile, la topographie, mode d'utilisation) et les 
coefficients d'abondance-dominance de chaque espèce.

Pour la caractérisation de la strate ligneuse dans chaque placette, des mesures dendrométriques classiques ont été portées sur tous les individus ligneux. Les diamètres ont été mesurés à l'aide de pied à coulisse pour les arbustes de petit diamètre et le compas forestier pour les arbres de gros diamètre, deux diamètres perpendiculaires du houppier et la hauteur totale ont été mesurés respectivement à l'aide de penta décamètre de $50 \mathrm{~m}$, et des jalons gradués. Les mesures ont porté sur les arbres de plus de $2 \mathrm{~cm}$ de diamètre considéré comme des individus adultes (Diouf et al., 2012).

\section{Analyse et traitement des données}

Les types biologiques de Raunkiaer (1934) utilisés pour la description de la flore de la commune de Dantchandou sont adaptés à l'étude de la végétation tropicale où la saison sèche correspond à la période défavorable. $\quad \mathrm{La} \quad$ distribution phytogéographique adoptée est celle de Guinochet (1973) utilisée dans les travaux sur la végétation de la sous-région (Mahamane, 2005 ; Ouédraogo, 2009 ; Morou, 2010).

\section{L'indice de diversité de Shannon (H en}

\section{bits) : Il est obtenu par la formule:}

avec $P_{i}=r_{i} / r$ où $r_{i}$ désigne le nombre $d$ 'individus d'une espèce $i$ et $r$ le nombre total d'individus dans la placette.

\section{L'indice d'équitabilité de Pielou (EQ) :}

Il est donné par la relation:

$$
H=-\sum_{i=1}^{s} p_{i} \log _{2} p_{i}
$$

$\mathrm{S}$ uesigne ia ricinesse specincue er $\log _{2} \mathrm{~S}$ la diversité maximale.

$$
\mathrm{Eq}=\frac{\mathrm{H}}{\mathrm{Hmax}} \text { avec } \mathrm{Hmax}=\log _{2} \mathrm{~S}
$$

\section{Densité des peuplements $\boldsymbol{N}$}

C'est le nombre moyen d'arbres de diamètre $\geq 2 \mathrm{~cm}$ par hectare. Elle est donnée par la formule :

$$
\mathrm{N}=\frac{\mathrm{n}}{\mathrm{s}}
$$

$\mathrm{n}=$ nombre total d'arbres des placettes de superficie $\mathrm{S}$

Le type phytogéographique adapté est celui de Guinochet utilisé dans les travaux sur la végétation de la sous-région. Les documents de base utilisés pour cette répartition phytogéographique sont : Flora of West Tropical Africa de Hutchinso et Daziel (1954, 1958, 1963, 1968 et 1972), la flore illustrée du Sénégal et la nouvelle flore illustrée du Sénégal et des régions voisines. Il s'agit de :

$\begin{array}{ll}- & \text { SZ : Soudano zambéziennes } \\ \text { - } & \text { GC : Guinéo congolaises ; } \\ \text { - } & \text { Sah.S : Saharo sindienne } \\ \text { - } & \text { Med : Méditerranéennes } \\ \text { - } & \text { i : Introduites }\end{array}$

\section{Distribution de Weibull des diamètres}

La distribution théorique de Weibull à trois paramètres (de position a, d'échelle ou de taille $b$ et de forme c) a été utilisé pour caractériser la structure des peuplements à la faveur de sa souplesse d'emploi et une grande variabilité de forme de distribution qu'elle produit. Le paramètre a correspond à la valeur seuil; c'est-à-dire la plus petite valeur de diamètre ; le paramètre $b$ est lié à la valeur centrale de la distribution des classe de diamètre. Enfin le paramètre $\mathrm{c}$ est lié à la structure observée et selon sa valeur conduit la distribution de Weibull à prendre plusieurs formes. Sa fonction de densité de probabilité $f$ $(x)$ se présente sous la forme ci-dessous :

$$
f(x)=\frac{c}{b}\left(\frac{x-a}{b}\right)^{c-1} \exp \left[-\left(\frac{x-a}{b}\right)^{c}\right]
$$

Un test d'ajustement de la distribution observée à la distribution théorique de Weibull (Rondeux, 1999) a été effectué a l'aide des logiciels Minitab16 et R 2.15.3. 


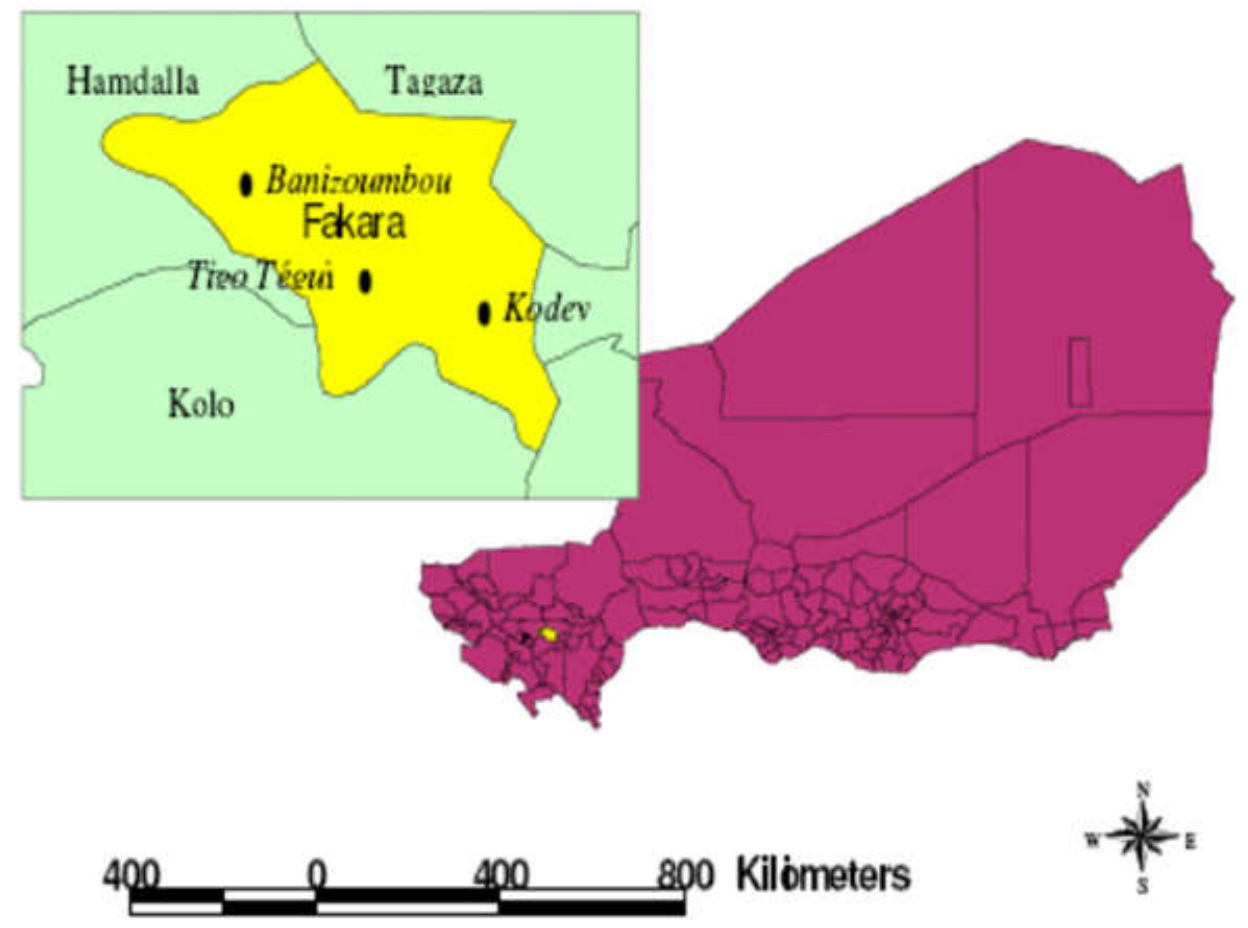

Figure 1: Carte de la situation géographique de la zone d'étude dans le département de Kolo (Niger). Source des données ILRI.

\section{RESULTATS}

\section{Caractéristiques globales de la flore}

Un total de 151 espèces végétales a été recensé dont 125 espèces herbacées et 26 espèces ligneuses réparties dans 101 genres et 28 familles (Figure 2). Le coefficient générique est donné par le rapport du nombre des genres à celui des espèces donc $105 / 151=0,69$ soit $69,53 \%$. Ce pourcentage de $69,53 \%$ traduit que la flore étudiée est riche.

\section{Analyse des formes biologiques globales}

Il ressort de l'analyse des résultats, la prédominance des thérophytes avec 66\% suivies des Phanérophytes $17 \%$ et Chamephytes avec 6\%. Par contre les géophytes, les hydrophytes et les hémicryptophytes sont très peu représentées avec $4 \%$ chacune. La prédominance des thérophytes témoigne l'aridité de la zone d'étude (Figure 3).

\section{Analyse des types phytogéographiques globaux}

La Figure 5 présente la distribution chorologique des espèces de la flore de commune de Dantchandou par fréquences d'apparition. Il ressort de l'analyse de cette figure que pour l'affinité chorologique, la distribution révèle au niveau global, la prédominance des espèces Guinéocongolaises et Soudoano-Zambésiennes (GCSZ) $36 \%$ suivies des espèces SoudanoZambéziennes (SZ) $32 \%$. Les espèces Soudano-Zambéziennes et Saharo-Sindiennes (SZ-Sah.S) représentent 17\%. Les espèces introduites (i) sont très peu représentées avec $1 \%$ (Figure 4 ).

Distribution des différents relevés en fonction des paramètres environnementaux Trois grands groupes des relevés se dégagent. Ces groupes correspondent aux 
différents types d'occupation des terres les plus fréquents dans la zone d'étude (Figure 5). En effet, les jachères se présentent comme des zones pastorales. Les vallées sont caractérisées par les champs. Les plateaux sont caractérisés par les brousses tigrées régulières et dégradées. Ces plateaux sont aussi exploités comme sources des bois énergies.

\section{Caractéristiques écologiques des formations végétales de champs}

Les relevés de végétation effectués dans les champs ont permis de recenser 106 espèces végétales réparties dans 81 genres et 23 familles. Les familles les plus représentées sont les Poaceae (20 espèces soit $18 \%$ ), les Leguminoseae-papilionaceae (10 espèces soit 9\%), les Convolvulaceae et les Cyperaceae avec chacune ( 9 espèces soit $8 \%)$, les Cucurbitaceae ( 8 espèces soit $7 \%$ ), les Amarantaceae ( 7 espèces soit $5 \%$ ) et enfin les autres familles les moins représentées se regroupent dans $45 \%$. L'indice de diversité de Shannon H est de 5,08 bits pour un indice de diversité maximale théorique (Hmax) de 6,78 bits, la régularité $\mathrm{R}$ est de 0,75 . Ces valeurs permettent de conclure que la diversité est bien élevée. La forme de vie la plus abondante en termes des spectres bruts dans les champs est celle des Thérophytes avec 59 espèces $(55,66 \%)$. Elle est suivie de celle des lianes thérophytes avec 15 espèces soient $14,15 \%$ et les microphanérophytes 9 espèces $(8,49 \%)$. Les autres formes telles que les géophytes rhizomateux, les hydrophytes, les nanophanérophytes, les lianes microphanérophytes, les hémicryptophytes, etc. présentent des très faibles taux d'apparition. La flore des champs est caractérisée par l'abondance des espèces Soudano-zambéziennes (SZ) avec 36 espèces, soit 33\%, les Guinéo-congolaise et Soudanozambézienne (GC-SZ) avec 35 espèces soit $32 \%$, les Guinéo-congolaise, Soudano- zambézienne et Saharo Sindienne (GC-SZSah.S) et les Soudano-zambézienne-Saharo Sindienne (SZ-Sah.S) avec chacune 18 espèces, soit $17 \%$. L'importance numérique des espèces $\mathrm{SZ}$ et GC-SZ montre que la flore des champs est caractérisée par l'abondance des espèces étrangères.

\section{Caractéristiques écologiques des formations végétales des jachères}

Dans les jachères, il a été recensé 17 familles réparties dans 47 genres. Les résultats montrent que la flore des jachères est dominée par des espèces annuelles telles que : les Poaceae (17 especes, 16 genres soit 16\%), les Léguminoseae-Papilionoideae (12 espèces 10 genres, soit $10 \%)$, les Convolvulaceae (8 espèces, 7 genres soit $6 \%$ ), les Cyperaceae (6 espèces, 5 genres, soit $6 \%$ ), les Amaranthaceae ( 5 espèces, 5 genres, soit $5 \%$ ), les Cucurbitaceae ( 5 espèces, 3 genres soit $5 \%$ ) et les Rubiaceae ( 5 espèces 3 genres, soit $5 \%$ ), les autres familles les moins représentées occupent un taux de 45\%. L'indice de diversité de Shannon H est de 5,16 bits avec une régularité $\mathrm{R}$ de 0,77 calculée à partir de l'indice de la diversité maximale théorique (Hmax) de 6,67 bits. Ces valeurs expliquent une diversité élevée dans les jachères. Les jachères sont caractérisées par une abondance de Thérophytes (57,42\%), des lianes Thérophytes $(10,89 \%)$ et des microphanérophytes $\quad(10,89 \%)$ Les chaméphytes, les nanophanérophytes, les Hydrophytes et les géophytes etc. constituent les types biologiques les moins représentés. L'analyse des types phytogéographiques des jachères révèle une abondance d'espèces soudano-zambéziennes (SZ) avec 38 espèces soit $36 \%$, suivies d'espèces GC-SZ (33 espèces soit $34 \%$ ), les espèces SZ-Sah.S sont représentées par 19 espèces soit $18 \%$, et d'espèces GC-SZ.-Sah.S avec 12 espèces soit $12 \%$. 


\section{Caractéristiques écologiques des formations végétales des plateaux}

Sur les plateaux, il a été recensé 111 espèces regroupées dans 24 familles. La famille des Poaceae est la plus représentée (19 espèces soit17\%) suivie de celle des Leguminoseae-Papiliniodeae (12 espèces, soit $11 \%$ ), les Cypéraceae et les Rubiaceae avec chacune $(8$ espèces soit $7 \%)$, les Cucurbitaceae (6 espèces soit $6 \%$ ) les Convolvulaceae (7especes soit 6\%), les Amanrethaceae et les Malvaceae avec chacun ( 5 espèces soit $5 \%$ ) et enfin les autres familles représentées en des très faibles proportions. L'indice de diversité de Shannon $\mathrm{H}$ est de 5,05 bits et une régularité $\mathrm{R}=0,73$ calculé à partir de Hmax (diversité maximale) de 6,94 bits. Ces valeurs démontrent une grande diversité spécifique et qu'il y a beaucoup d'espèces qui se partagent à peu près le même recouvrement. $\mathrm{La}$ prédominance de Thérophytes (59,45\%) est suivie ordre d'importance des micro phanérophytes $(10,81 \%)$, des Lianes thérophytiques avec $9,90 \%$, les Chamephytes 4,50\%, des géophytes et des nanophanérophytes, avec chacune 3,6\%. Les autres formes sont représentées en des très faibles proportions. Le spectre des types phytogéographiques de la Figure 4 indique une dominance d'espèces Guinéo-Congolaise-Soudano-zambiennes (GC-SZ) $\quad 36 \%$ suivies dans l'ordre d'importance par des espèces SoudanoZambéziennes (SZ) 31\%, les espèces Soudano-Zambézienne-Saharo-Sindiennes (SZ-Sah .S) 18\%, les espèces GuinéoCongolaise-Soudano-zambiennes-SaharoSindiennes GC-SZ-Sah.S (14\%). Les espèces introduites sont très peu avec moins de $1 \%$ de la fréquence totale.

\section{Caractéristiques dendrométriques}

La densité globale sur pieds est en moyenne de 745,2 individus/ha; la plus forte densité (1020 individus/ha) est obtenue sur les plateaux où sont localisées les brousses tigrées régulières et dégradées. La plus faible densité est enregistrée dans les champs de culture (129,2 individus/ha), tandis qu'elle est de 385,5 individus/ha dans les jachères. Le diamètre moyen du houppier, le diamètre moyen des arbres sur pied sont respectivement de $2,13 \pm 1,03$ m et $4,56 \pm 3,98 \mathrm{~cm}$ sur les plateaux, de $1,85 \pm 1,71 \mathrm{~m}$ et $8,92 \pm 12,8 \mathrm{~cm}$ dans les champs et enfin de $1,98 \pm 1,06 \mathrm{~m}$ et 4,69 $\pm 6,07 \mathrm{~cm}$ dans les jachères. L'analyse du recouvrement ligneux par type d'occupations de terres montre que les plateaux ont le plus fort recouvrement avec $40,53 \%$ et viennent après, les jachères avec 15,96 puis les champs de culture avec $6,96 \%$ (Tableau 1 ).

\section{Structure en diamètre}

La structure en diamètre de la végétation est présentée à la Figure 6 On note au niveau global que l'ensemble des formations végétales présente une allure en "J Renversée" caractéristique des peuplements multi-spécifiques avec la valeur de paramètre de forme, c inférieur à 1 . Les individus de diamètres compris entre 3 et $15 \mathrm{~cm}$ sont les plus fortement représentés, tandis que ceux dont les diamètres sont supérieurs à $33 \mathrm{~cm}$ y sont quasi-absents. La même allure en "J Renversée' est observée au niveau des formations du plateaus, champs et jachères. Par contre les formations des premières auréoles du village ont une valeur de paramètre de forme $\mathrm{c}$, égale à 1,043 . Cette structure révèle plutôt une distribution asymétrique positive, caractéristique des peuplements avec prédominance d'individus jeunes ou de faibles diamètres (Figure 6). $\mathrm{La}$ prédominance des individus jeunes s'explique par la dominance des espèces multicaules dont entre autres Combretum micranthum et surtout Guiera senegalensis. 


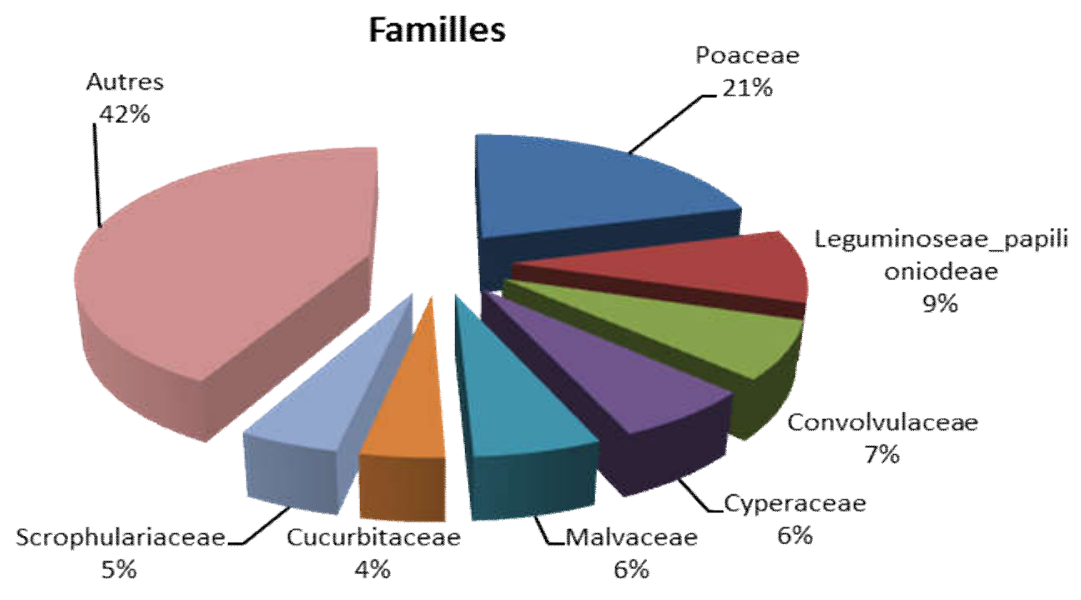

Figure 2:Composition floristique de la commune de Dantchandou.

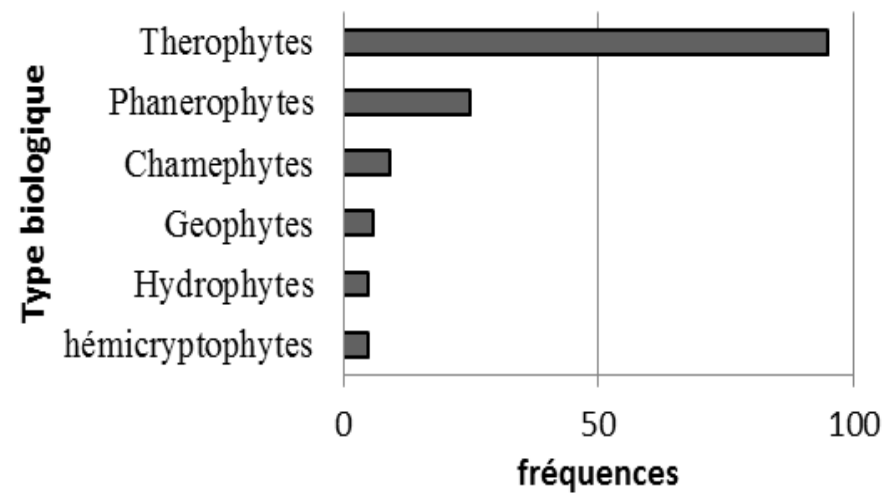

Figure 3: Spectre brut de types biologique de la commune de Dantchandou.

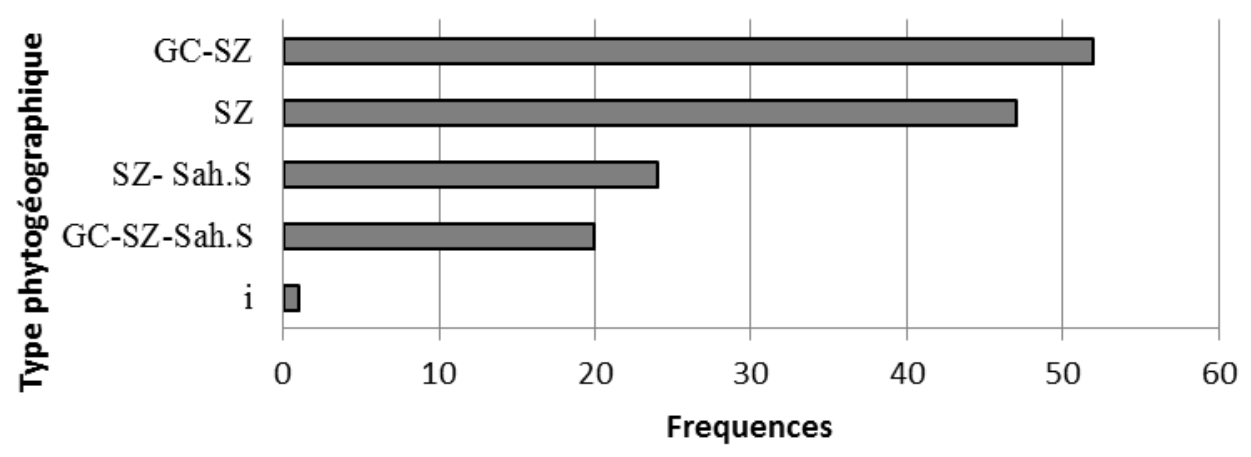

Figure 4 : Spectre de type phytogéographique de la commune de Dantchandou. 


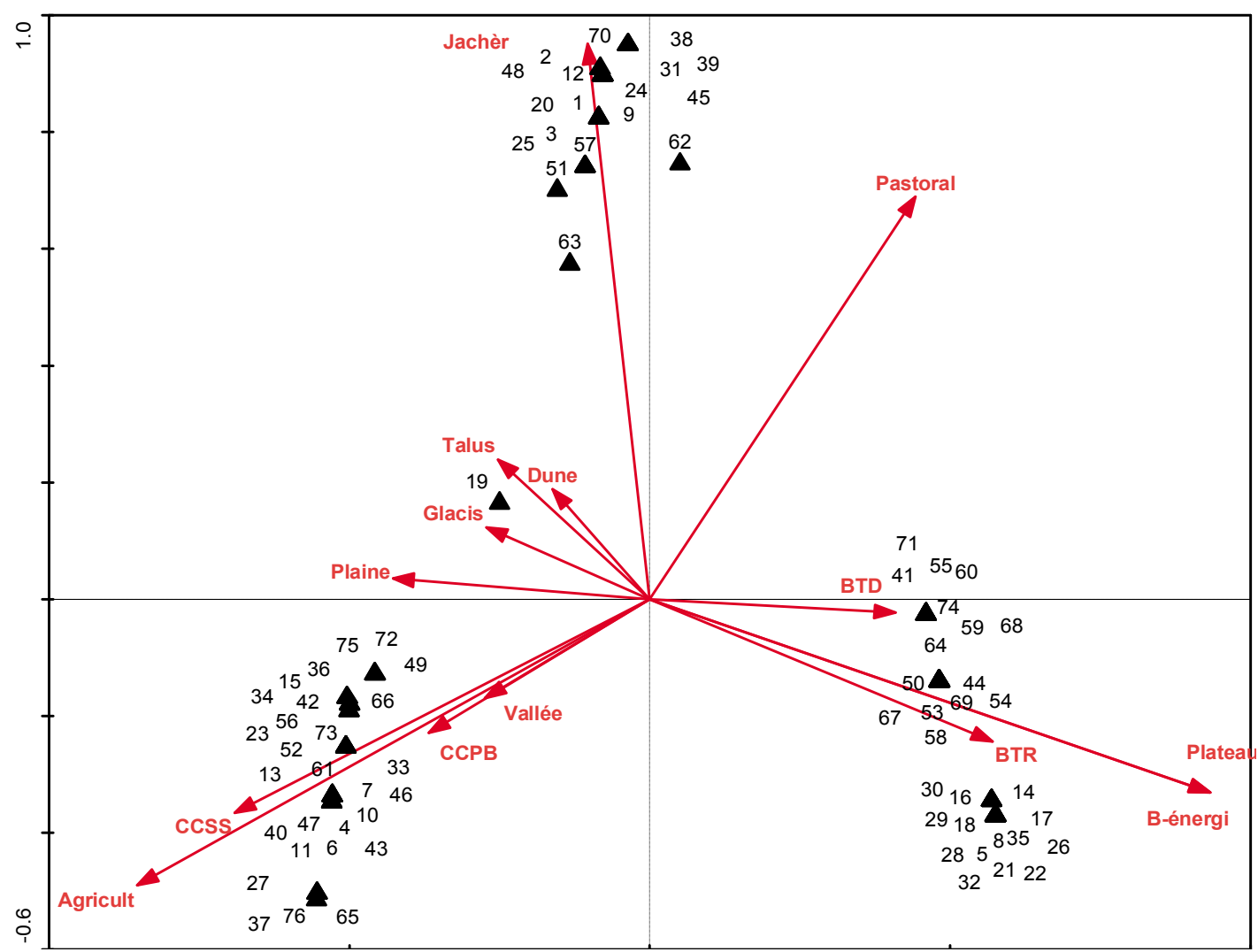

Figure 5: Répartition des relevés en fonction des types d'occupations des terres et les paramètres stationnaires.

Tableau 1 : Paramètres dendrométriques des différents types d'occupations des terres.

\begin{tabular}{lccccc}
\hline Unités & $\begin{array}{c}\text { Densité } \\
\text { moyenne } \\
\text { (individus/ha) }\end{array}$ & $\begin{array}{c}\text { Diamètre moyen } \\
\text { du houppier (m) }\end{array}$ & $\begin{array}{c}\text { Recouvrement } \\
\text { moyen (\%) }\end{array}$ & $\begin{array}{c}\text { hauteur } \\
\text { moyenne } \\
\mathbf{( m )}\end{array}$ & $\begin{array}{c}\text { Diamètre } \\
\text { moyen (cm) }\end{array}$ \\
\hline plateaux & 1020 & $2,13 \pm 1,03$ & 40,53 & $3,86 \pm 1,2$ & $4,56 \pm 3,98$ \\
champs & 129,2 & $1,85 \pm 1,71$ & 6,98 & $4,95 \pm 2,23$ & $8,92 \pm 12,8$ \\
jachères & 385,5 & $1,98 \pm 1,06$ & 15,96 & $4,31 \pm 1,24$ & $4,69 \pm 6,07$ \\
\hline
\end{tabular}



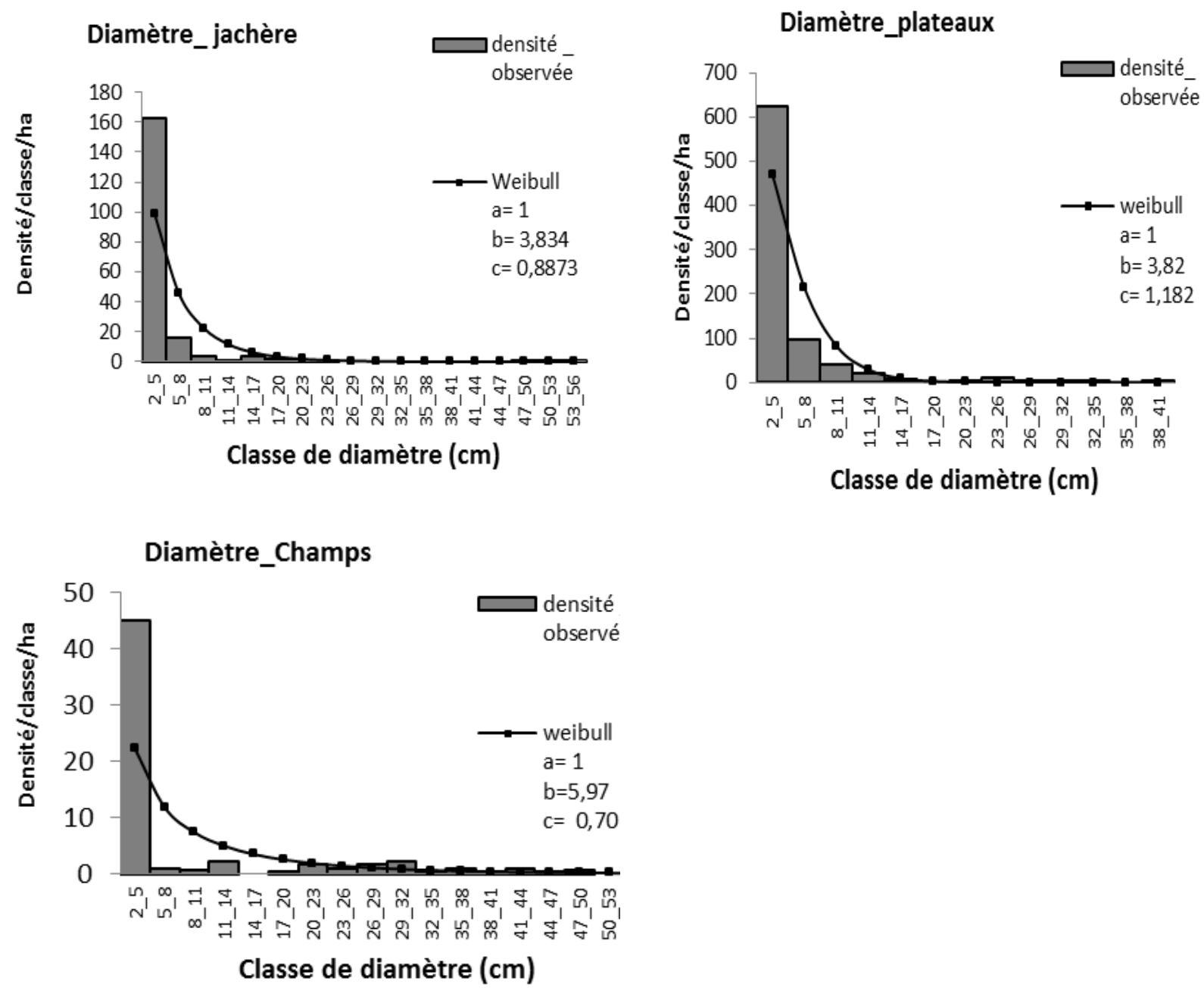

Figure 6 : Structure en diamètre des différents types d'occupations des terres dans la commune de Dantchandou.

\section{DISCUSSION}

Les familles les plus représentées de la végétation de la commune de Dantchandou sont les Poaceae (26 genres, 32 espèces, soit 21\%), les Leguminoseae-papilioniodae (9 genres 14 espèces, soit $9 \%$ ), les Convolvulaceae (8 genres, 9 espèces, soit 7\%), les Cyperaceae (6 genres, 9 espèces, soit $6 \%$ ), les Malvaceae, (6genres, 8 espèces, soit $6 \%$ ), les Scrophulariaceae, (5genres, 7 especes soit 5\%), les Cucurbitaceae, (3 genres , espèces, soit $4 \%$ ). Ces résultats corroborent ceux trouvés par Saadou (1990), selon qui, les Poaceae (168 espèces) prédominent suivies des Leguminoseae-Papilionoideae (106 espèces), des Malvaceae (31 espèces), des Convolvulaceae (31 espèces) et des Euphorbiaceae (25 espèces). Pour Mahamane (2005) l'ordre de dominance est les Poaceae (218 espèces soit 43\%), suivies des Leguminosae-Papilionoideae $(144$ espèces soit 28,4\%) dans le Parc W. Quant à Morou (2010), l'ordre de dominance est les Poaceae (34 espèces soit 37,7\%) suivies de l'ordre de 
Leguminoseae-Papilionoideae (18 espèces soit $20 \%$ ) et Leguminoseae-Mimosoideae (15 espèces soit $16 \%$ ) dans la brousse tigrée de Kouré. Les différences dans l'ordre des familles seraient liées à la différence des biotopes explorés par ces auteurs.

En effet, Saadou (1990) a caractérisé la végétation des terres fermes, Mahamane (2005) a décrit les groupements végétaux du Parc W (protection) et Morou, ceux de l'habitat de la girafe dans la commune rurale de Dantchandou. Cette diversité biotopiques induit de facto celle de la flore du pays que Saadou (1990) estime à 1500 espèces. Pour ce qui est de la répartition phytogéographique, la flore est à prédominance des espèces soudanozambézienne et des espèces Guinéo congolaises et soudan-zambézienne. Les mêmes résultats sont observés au niveau des plateaux. Quant aux champs et jachères, l'ordre est: la prédominance des espèces soudano-zambézienne suivies des espèces Guinéo congolaises- soudano-zambézienne. Cela démontre le caractère moyen humide de certaines stations et xérique des autres. La faible diversité des groupements des formations des plateaux relèverait d'une importante pression anthropique sur ces formations et un bilan hydrique défavorable dû à la mauvaise infiltration. Ichaou (2000) a estimé en moyenne 60000 ha la superficie soustraite des formations contractées des plateaux du Niger annuellement du fait des défrichements agricoles et de l'exploitation incontrôlée des ressources ligneuses.

Le plus grand recouvrement est observé au niveau des plateaux et faible au niveau des champs et des jachères. Ces résultats peuvent s'expliquer par l'aspect jointif des branchages dans les brousses tigrées régulières du fait de la très forte densité des ligneux. Donc l'enchevêtrement entre différents individus concourt à l'augmentation de recouvrement des espèces. Par contre dans les champs et les jachères l'activité de défrichement entraine la coupe des plus grands arbres et occasionne le faible recouvrement observé.

La brousse tigrée qui constitue la majeure partie de la formation végétale de la commune de Dantchandou se situe également dans le bassin d'approvisionnement en bois de la ville de Niamey (capitale du Niger) dont les besoins en bois énergie vont en augmentant (Mahamane et al., 2007). Ce prélèvement important serait à la base de la disparition de plusieurs espèces végétales aussi bien ligneuses que herbacées. Un autre facteur de dégradation du couvert végétal est le déficit pluviométrique de ces dernières années qui provoque le dépérissement des essences forestières sensibles entrainant du coup une contraction plus importante des bandes de végétation au profit des bandes de sols nus (Ichaou, 2000). L'impact de ces différents paramètres sur la diversité biologique et la structure du peuplement ligneux se traduit par une fragmentation de l'habitat et une réduction de la diversité intra-biotique. Cette fragmentation correspond à l'apparition des nouveaux types d'occupations des terres et constitue une menace à la biodiversité et à la conservation des espèces (Bogaert et Hong, 2004).

La zone de Dantchandou présente de manière générale les zones de plateaux à brousse tigrée des champs et des jachères. La végétation naturelle est une végétation proche de la formation à Combretum glutinosum et Guiera senegalensis sur sols gréseux décrite par White (1986) dans la zone de transition régionale du Sahel. Dans tous les milieux post-culturaux de la région, l'arbuste Guiera senegalensis domine strictement la strate ligneuse, d'ailleurs extrêmement pauvre. C'est seulement dans les stations les plus humides qu'ils sont remplacés par Piliostigma reticulatum, Combretum glutinosum, Annona senegalensis ou Hyphaene thebaica (Fournier et al., 2001). Durant les cinq premières années 
d'abandon cultural, les ligneux qui étaient présents sous forme de rejets des souches lors de la mise en jachère augmentent en taille, mais il n'y a que peu de recrutement. Au-delà de cinq ans, taille et phytovolume diminuent, témoignant du vieillissement de la population. La flore herbacée, pauvre elle aussi, est dominée par un groupe d'espèces ubiquistes (Zornia glochidiata, Eragrostis tremula, Mitracarpus scaber) pendant toute la phase post-culturale. Une dynamique successionnelle existe cependant: vers quatre à cinq ans, plusieurs familles sont bien représentées, puis ce sont les graminées qui dominent avec apparition d'espèces de grande taille (Aristida spp., Ctenium elegans et Andropogon gayanus); plus tard enfin s'affirme la légumineuse Zornia glochidiata. Les graminées pérennes, représentées presque exclusivement par Andropogon gayanus restent très peu abondantes, même aux stades âgés. En fait, la plupart des vieilles jachères correspondent à un état dégradé par l'Homme, où la dynamique de succession ne s'exprime qu'imparfaitement. Des observations analogues ont été faites sous un climat semblable à Dalombedougou au Mali et dans le delta intérieur du Niger (Hiernaux et Le Houerou, 2006).

L'analyse de la structure d'ensemble en diamètre des peuplements ligneux de la commune de Dantchandou (toutes espèces confondues) présente une distribution en « $\mathrm{J}$ renversé » et s'ajuste globalement à la distribution théorique de Weibull avec le paramètre de forme, $\mathrm{c}$ prenant une valeur de 0,95 . Cette structure est caractéristique des peuplements naturels mono-spécifiques avec prédominance d'individus jeunes ou de faible diamètre. Quel que soit le site, plus de 95\% des individus ont un diamètre inférieur à $5 \mathrm{~cm}$. Le très faible pourcentage des individus à diamètre supérieur à $5 \mathrm{~cm}$ pourrait être lié à la coupe du bois énergie dans la zone. Les densités élevées des classes de faible diamètre assurent l'avenir de la formation naturelle tandis que les faibles densités des classes de gros diamètre résultant de la sélection sont des semenciers qui assurent la pérennité du peuplement à travers la production de graines. La maîtrise de la structure en diamètre des ligneux est nécessaire pour tout aménagement des peuplements forestiers. Ouédraogo et al. (2006), ont également observés la même structure respectivement dans les galeries forestières au Bénin et au Burkina-Faso (marre des Hippopotames).

Les paramètres de forme $\mathrm{c}$ de peuplement des jachères et champs présentent des valeurs inferieures à 1 , ces structures semblent être normales c'est-à-dire des peuplements en pleines régénérations. Par contre ceux du plateau et de la première auréole du village présente des valeurs supérieures à 1. Cela suggère une perturbation dans la structure en diamètre de peuplement $\mathrm{du}$ plateau et première auréole de village. L'explication réside évidement dans la recherche de bois de chauffe par les femmes dans les environnements immédiats de village. Quant au plateau, ils font l'objet d'une exploitation commerciale de bois en direction de Niamey. Sur le plateau, la prédominance des individus jeunes semble être une structure normale, mais en réalité c'est une structure forcée car les individus de gros diamètre sont systématiquement coupés. Pour le recouvrement, ce sont les formations des brousses tigrées qui prennent le dessus, par la particularité de la végétation du plateau qui se contracte en bandes boisées.

\section{Conclusion}

L'analyse globale de la composition floristique a mis en évidence l'impact de l'occupation des terres sur la dynamique de la strate herbacée et ligneuse. La forte représentativité des thérophytes traduit particulièrement les conditions écologiques sévères qui caractérisent le milieu. Les formes 
de vie les plus favorisées sont celles capables de boucler leur cycle en une saison. Au point de vue chronologique, l'étude fait apparaître une prépondérance des espèces guinéo congolaises- soudano-zambéziennes. Les espèces Soudano-zambézienne Saharo Sindienne sont sous représentées. Ces résultats montrent que le déterminisme des espèces vivant dans la zone n'est pas essentiellement d'ordre climatique. $\mathrm{La}$ diversité alpha varie d'un type de formation à l'autre et au sein d'un même type de formation. Elle est plus importante dans les agrosystèmes en particulier dans les champs et les jachères. Alors que l'attention des gestionnaires et du public se focalise depuis toujours sur les ligneux, en fait ce sont les herbacées qui supportent l'essentiel de la richesse spécifique et des menaces. En conséquence, il faudrait assez vite orienter les efforts de conservation vers les plus sensibles d'entre elles.

\section{REFERENCES}

Bogaert J, Hong SK. 2004. Lands cape ecology: monitoring landscape dynamics using spatial pattern metrics. In Ecological Issues in a Changing World: Status, Response and Strategy, Hong SK, Lee JA, Ihm B-S, Farina A, Son Y, Kim E-S, Choe JC (eds). Kluwer Academic Publishers: Netherlands; 109131.

Braun-Blanquet J. 1932. Plant Sociology. The Study of Plant Communities. Ed. McGray Hill : London, New York ; 439 p.

Cheula A. 2009. Dynamique de l'occupation des sols en milieu sahélien. Espaces cultivés et couverture ligneuse dans la commune de Hombori, Mali. Master 2 Télédétection et géomatique appliquées à l'environnement, Paris 7, 44p.

Diouf A, Barbier N, Lykke AM, Couteron P, Deblauwe V, Mahamane A, Saadou M,
Bogaert J. 2012. Relationships between fire history, edaphic factors and woody vegetation structure and composition in a semi-arid savanna landscape (Niger, West Africa). Journal of Applied Vegetation Science, 15(4): 488-500.

FAO. 2003. Gestion de la fertilité des sols pour la sécurité alimentaire en Afrique subsaharienne. Rome, Italie, $63 \mathrm{p}$.

Fournier A, Hien M, Millogo-Rasolodimby J. 2001. Les jachères de moins de cinq ans en savane soudanienne: richesse floristique, structure de la végétation. Cas de Bondoukuy (Ouest du Burkina Faso). In Floret et Pontanier, 1: 390399.

Gounot M. 1969. Méthodes d'Etude Quantitative de la Végétation. Masson: Paris ; 314 pp.

Guinochet M. 1973. La Phytosociologie. Collection d'Ecologie I. Masson éd: Paris ; $227 \mathrm{p}$.

Hiernaux P, Le Houerou HN. 2006. Les parcours du Sahel. Sécheresse, 17(1-2): 51-71.

Ichaou A. 2000. Dynamique et productivité des principales structures forestières contractées des plateaux de l'Ouest nigérien. Thèse de Doctorat, Université Paul Sabatier de Toulouse III France (Laboratoire d'Écologie Terrestre), 230p.

Mahamane A. 2005. Etudes floristique, phytosociologique et phytogéographique de la végétation du Parc Régional du W du Niger. Thèse de Doctorat ès Sciences Agronomiques et Ingénierie Biologique. Université Libre de Bruxelles, 536 p.

Mahamane A, Ichaou A, Ambouta K, Saadou M, Morou B, Amani I, Mahamadou H, d'Herbès JM, Gineste P, Wata I. 2007. Indicateurs écologiques de la période optimale de remise en culture de jachères au Niger. Sécheresse, 18: 1-7.

Morou B. 2010. Impacts de l'occupation des sols sur l'habitat de la girafe au Niger et 
enjeux pour la sauvegarde du dernier troupeau de girafes de l'Afrique de l'Ouest, Thèse de Doctorat de $3 \mathrm{e}$ cycle de Biologie et Ecologie Végétales. Université Abdou Moumouni de Niamey, $198 \mathrm{p}$.

Ouédraogo A. 2006. Diversité et dynamique de végétation ligneuse de la partie orientale du Burkina Faso. Thèse de doctorat Université de Ouagadougou Burkina Faso. 195p + Annexe.

Ouédraogo A, Adjima T, Hahn-Hadjali K, Guinko S. 2006. Diagnostic de l'état de dégradation des peuplements de quatre espèces ligneuses en zone soudanienne du Burkina Faso. Sécheresse, 17(4): 485491.

Pascal F. 2010. Influences of anthropogenic activities on the giraffe (Giraffa camelopardalis) population of Omo National Park. Igwg-Giraffa, 3(1).

Raunkiaer C. 1934. The Life froms of Plante and Statiscal Plant Geography. Clarendon Press: Oxford, RoyaumeUnis ; 632p.

Rondeux J. 1999. La mesure des peuplements forestiers. Gembloux; Belgique; les presses agronomique de Gembloux; $522 \mathrm{p}$.

Saadou M. 1990. La végétation des milieux drainés nigériens à l'Est du fleuve Niger. Thèse de Docteur ès Sciences Naturelles, Université de Niamey, 395 p. + annexes.

White F. 1986. La végétation de d'Afrique. Mémoire accompagnant la carte de la végétation de l'Afrique. UNESCO/AETFAT/UNSO ; Orstom; $384 p$. 